The Astrophysical Journal, 655:332-341, 2007 January 20

(C) 2007. The American Astronomical Society. All rights reserved. Printed in U.S.A.

\title{
THE ABUNDANCE OF CARBON DIOXIDE ICE IN THE QUIESCENT INTRACLOUD MEDIUM
}

\author{
D. C. B. Whittet, ${ }^{1,2}$ S. S. Shenoy, ${ }^{1,3}$ E. A. Bergin, ${ }^{4}$ J. E. Chiar, ${ }^{5}$ P. A. Gerakines, ${ }^{6}$ \\ E. L. Gibb, ${ }^{7}$ G. J. Melnick, ${ }^{8}$ and D. A. Neufeld ${ }^{9}$ \\ Received 2006 June 9; accepted 2006 September 28
}

\begin{abstract}
We present new observations with the Infrared Spectrograph on board the Spitzer Space Telescope of the solid$\mathrm{CO}_{2}$ absorption feature near $15 \mu \mathrm{m}$ in the spectra of eight field stars behind the Taurus complex of dark clouds. Solid $\mathrm{CO}_{2}$ is detected in six lines of sight. New results are combined with previous data to investigate the correlation of $\mathrm{CO}_{2}$ column density with those of other major ice constituents $\left(\mathrm{H}_{2} \mathrm{O}\right.$ and $\left.\mathrm{CO}\right)$ and with extinction. $\mathrm{CO}_{2}$ is shown to display a "threshold extinction" effect, i.e., a minimum extinction $\left(A_{0}=4.3 \pm 1.0 \mathrm{mag}\right)$ required for detection, behavior similar to that previously reported for $\mathrm{H}_{2} \mathrm{O}$ and $\mathrm{CO}$. We find a particularly tight correlation through the origin between $N\left(\mathrm{CO}_{2}\right)$ and $N\left(\mathrm{H}_{2} \mathrm{O}\right)$, confirming that these species form in tandem and coexist in the same (polar) ice layer on the grains. The observed composition of the mantles is broadly consistent with the predictions of photochemical models with diffusive surface chemistry proposed by Ruffle \& Herbst. Comparison of our results for Taurus with published data for Serpens indicates significant differences in ice composition consistent with enhanced $\mathrm{CO}_{2}$ production in the latter cloud. Our results also place constraints on the distribution of elemental oxygen between ices and other potential reservoirs. Assuming a constant $N(\mathrm{H})$ to extinction ratio, we show that $\sim 65 \%$ of the solar $\mathrm{O}$ abundance is accounted for by summing the contributions of ices $(\sim 26 \%)$, refractory dust $(\sim 30 \%)$ and gas-phase $\mathrm{CO}(\sim 9 \%)$. If the Sun is an appropriate standard for the interstellar medium, the "missing" oxygen may reside in atomic O I gas and/or (undetected) $\mathrm{O}_{2}$ within the ices.
\end{abstract}

Subject headings: dust, extinction — ISM: abundances — ISM: individual (Taurus Dark Cloud) — infrared: ISM: lines and bands - ISM: molecules

\section{INTRODUCTION}

Carbon dioxide $\left(\mathrm{CO}_{2}\right)$ has proven to be a common constituent of the ice mantles that accumulate on the surfaces of dust grains in dense interstellar clouds. Solid $\mathrm{CO}_{2}$ absorption has been detected in the infrared spectra of young stellar objects (YSOs) observed with both the Infrared Space Observatory (ISO; e.g., de Graauw et al. 1996; d'Hendecourt et al. 1996; Gerakines et al. 1999; Nummelin et al. 2001; Gibb et al. 2004) and the Spitzer Space Telescope (e.g., Boogert et al. 2004; Watson et al. 2004; Dartois et al. 2005; Pontoppidan et al. 2005). The strength of the absorption is consistent with an abundance in the range $9 \%-37 \%$ relative to $\mathrm{H}_{2} \mathrm{O}$, the dominant ice constituent. This result is important, not only because the presence of $\mathrm{CO}_{2}$ places constraints on chemical models for mantle formation and evolution, but also because it provides an empirical technique for investigating the thermal evolution of the ices in the environs of the YSOs. The profile shape of the absorption feature associated with the $\mathrm{O}=\mathrm{C}=\mathrm{O}$ vibrational bending mode, near $15 \mu \mathrm{m}$ in wavelength, has been shown to be especially sensitive to the thermal history and structure of the ice mixture, displaying subfeatures on crystallization that are diagnostic of annealing temperature and segregation of constituents (Ehrenfreund et al. 1999; Dartois et al. 1999; van Broekhuizen et al. 2006).

\footnotetext{
1 Department of Physics, Applied Physics and Astronomy, Rensselaer Polytechnic Institute, Troy, NY.

2 On leave at Center for Imaging Science, Rochester Institute of Technology, Rochester, NY.

3 Current address: Spitzer Science Center, California Institute of Technology, Pasadena, CA.

4 Department of Astronomy, University of Michigan, Ann Arbor, MI.

5 SETI Institute, Mountain View, CA.

6 Department of Physics, University of Alabama, Birmingham, AL.

7 Department of Physics and Astronomy, University of Missouri, St. Louis, MO.

8 Harvard-Smithsonian Center for Astrophysics, Cambridge, MA.

9 Department of Physics and Astronomy, Johns Hopkins University, Baltimore, MD.
}

In order to provide a firm basis for interpretation of $\mathrm{CO}_{2}$ features in YSO spectra, it is necessary to establish the nature of $\mathrm{CO}_{2}$-bearing ices in the cold, quiescent regions of molecular clouds, i.e., in the "intracloud medium" (ICM), remote from embedded sources. Key properties include abundance relative to other ice constituents and dust extinction, and the nature of the molecular environment in which the $\mathrm{CO}_{2}$ typically resides within the mantles. Answering these questions may, in turn, help us to distinguish between possible mechanisms for the origin of $\mathrm{CO}_{2}$, such as catalytic grain-surface reactions and photochemical reactions driven by external radiation fields. The traditional method used to investigate the dusty/icy components of molecular clouds is by observation of solid state absorption features in the spectra of background field stars. However, because these objects are intrinsically faint in the mid-infrared, very few could be observed with the ISO short-wavelength spectrometer (SWS): the sample for the solar neighborhood of the Galaxy was limited to two latetype giants behind the Taurus dark cloud (Whittet et al. 1998; Nummelin et al. 2001). ${ }^{10}$ Having much greater sensitivity, and with coverage of the $15 \mu \mathrm{m} \mathrm{CO}$ feature in its high-resolution mode, Spitzer's Infrared Spectrograph (IRS) is well suited to addressing this problem. First results were published recently by Bergin et al. (2005) and Knez et al. (2005). In this paper, we report new spectra for eight field stars background to the Taurus dark cloud, and present a detailed analysis of all relevant results available to date.

\section{OBSERVATIONS AND DATA REDUCTIONS}

The observations reported here were obtained as part of the Spitzer Space Telescope Cycle 1 General Observer (GO) program 3303. All observations were made between 2004 October

\footnotetext{
${ }^{10}$ Solid $\mathrm{CO}_{2}$ was also detected by $I S O$ in a molecular cloud (or clouds) foreground to the infrared sources near the Galactic center (Chiar et al. 2000), but the nature and locus of the absorbing region is poorly constrained.
} 
TABLE 1

OBSERVATIONS

\begin{tabular}{|c|c|c|c|c|c|}
\hline Source $\mathrm{ID}^{\mathrm{a}}$ & Association & AOR Key & Date of Observation & Plot Label (Fig. 1) & $\begin{array}{c}\int \tau(\nu) d \nu^{\mathrm{b}} \\
\left(\mathrm{cm}^{-1}\right)\end{array}$ \\
\hline 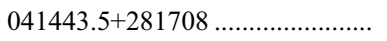 & & 10717696 & 2005 Feb 9 & (d) & $2.7 \pm 0.8$ \\
\hline $042630.7+243637$............................. & & 10719744 & 2005 Feb 9 & (c) & $3.0 \pm 0.5$ \\
\hline 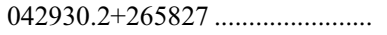 & & 10720256 & 2005 Feb 9 & (h) & $1.9 \pm 0.4$ \\
\hline 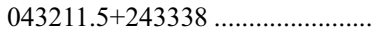 & Elias 9 & 10720512 & 2004 Oct 4 & (a) & $<0.5$ \\
\hline 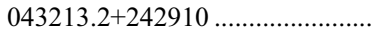 & & 10720768 & 2005 Feb 9 & $(g)$ & $3.6 \pm 0.5$ \\
\hline 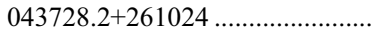 & Tamura 2 & 10721792 & 2005 Feb 14 & (e) & $1.0 \pm 0.5$ \\
\hline $043926.9+255259 \ldots \ldots \ldots \ldots \ldots \ldots \ldots$ & Elias 15 & 10722304 & 2005 Feb 14 & (b) & $2.5 \pm 0.4$ \\
\hline $044124.7+255448 \ldots \ldots \ldots \ldots \ldots \ldots$ & HD 283809 & 10723072 & 2005 Mar 11 & $(f)$ & $<0.7$ \\
\hline
\end{tabular}

${ }^{\text {a }}$ The identification number denotes equatorial J2000 coordinates in format hhmmss.s + ddmmss.

${ }^{\mathrm{b}}$ Values determined from our observations of the $15.3 \mu \mathrm{m}$ bending-mode absorption feature of solid-phase $\mathrm{CO}_{2}$.

and 2005 March with the IRS (Houck et al. 2004) in shortwavelength, high-resolution staring mode, covering the spectral range $10.0-19.5 \mu \mathrm{m}$ at a resolving power $\lambda / \Delta \lambda \approx 600$. The sources observed are listed in Table 1 together with the Astronomical Observation Request (AOR) key, which provides unique identification of each spectrum in the Spitzer archive.

The data were processed with the Spitzer Science Center (SSC) pipeline S13.2.0, which provides basic calibrated data (BCD). Fringing sometimes evident in spectra from earlier versions of the pipeline are effectively removed by the flat-fielding process in this version. Examination of BCD images revealed that some pixels were unusable "rogue" or "hot" pixels (the latter attributed to cosmic-ray hits). We identified these bad pixels and created a pixel mask image using IRSCLEAN_MASK, an interactive IDL tool. This was used to mask out the bad pixels from the BCD image. Further analysis of the cleaned BCD was carried out using the IRS team's SMART program (Higdon et al. 2004). To provide redundancy against cosmic-ray hits and detector artifacts, each source was placed at two slit location (nods) along the slit axis for each ramp. The BCD, uncertainty, and mask images were combined into a three-plane image and were used as input for SMART. Full-slit extractions were performed for each nod position. Wavelength calibration was performed using the calibration table provided by the SMART developer team. Overlapping spectral orders that contained the poorly calibrated long-wavelength part of each order were deleted. This is an important factor in obtaining reliable $15 \mu \mathrm{m} \mathrm{CO}_{2}$ profiles, as two orders overlap in this spectral region. For accurate flux calibration we used the standard star HR 6688 (K2 III) and an extraction procedure similar to that described above. Each target spectrum was ratioed, nod position by nod position, with the spectrum of the standard star and multiplied by a template spectrum appropriate for HR 6688 from Decin et al. (2004). Spectra from all nod positions were averaged to obtain the final calibrated source spectrum. Results for all sources are shown in Figure 1. The weak apparent feature near $14.2 \mu \mathrm{m}$ seen in several spectra is thought to be not real but an artifact of stitching orders.

Continua were determined for each flux spectrum by secondorder polynomial fitting to wavelength regions adjacent to the $\mathrm{CO}_{2}$ feature (13.5-14.9 and 16.1-17.5 $\mu \mathrm{m}$ ). The adopted continua are shown superposed on the spectra in Figure 1. These were used to convert flux to optical depth (Fig. 2).

\section{PROFILE SHAPE AND COLUMN DENSITY}

Perusal of our spectra (Fig. 1) indicates that $\mathrm{CO}_{2}$ is clearly detected in six out of eight lines of sight. The profile shape and
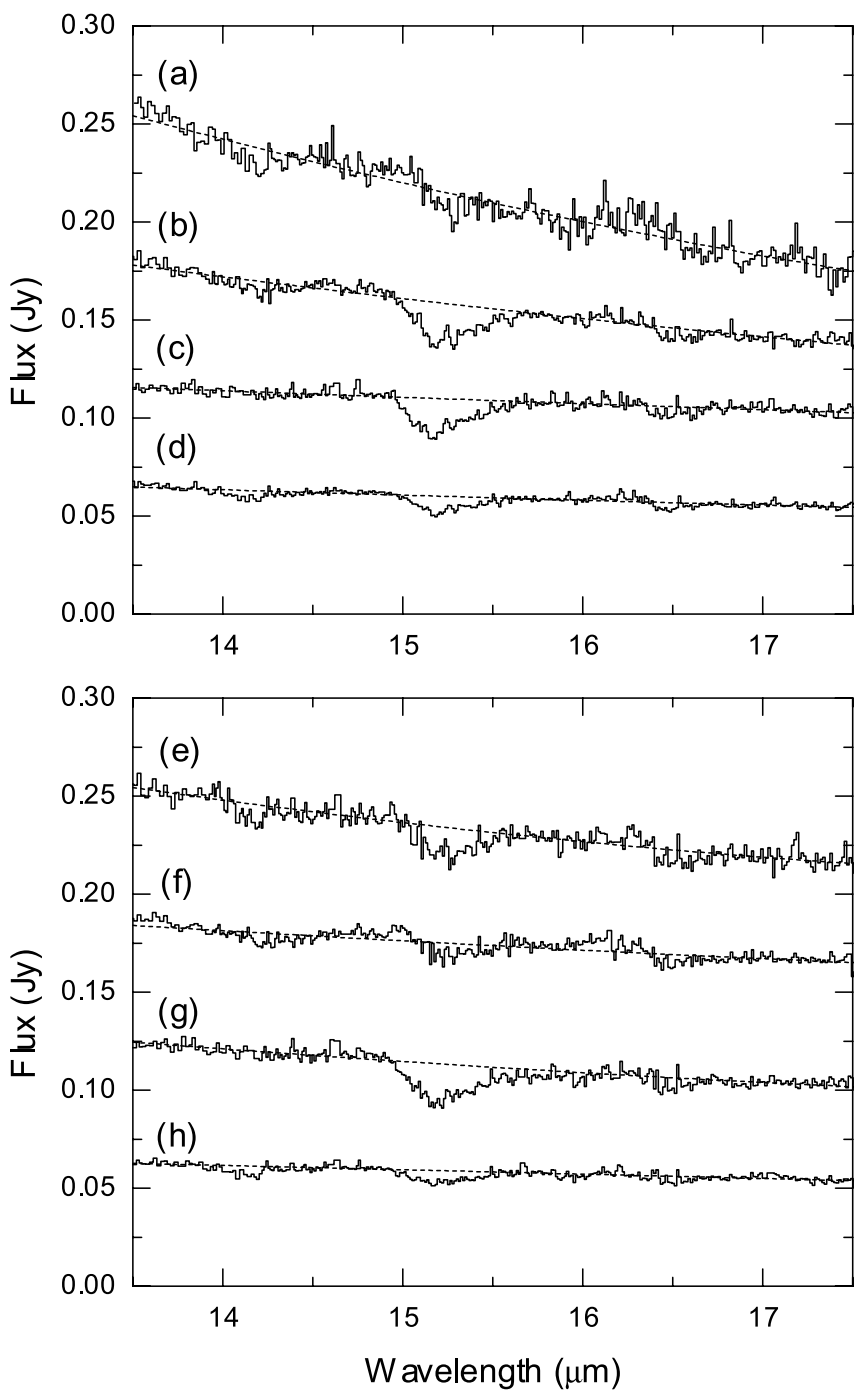

FIG. 1. - Spectra. Top panel: (a) $043211.5+243338(\times 1)$; (b) $043926.9+$ $255259(\times 1.2)$; (c) $042630.7+243637(\times 1.2)$; and $(d) 041443.5+281708(\times 0.9)$. Bottom panel: $(e) 043728.2+261024(\times 2.2) ;(f) 044124.7+255448(\times 1.2)$; (g) $043213.2+242910(\times 1.4)$; and $(h) 042930.2+265827(\times 1)$. The numbers in parentheses are scaling factors applied for clarity of display. The dashed lines are the adopted continua (see text). 


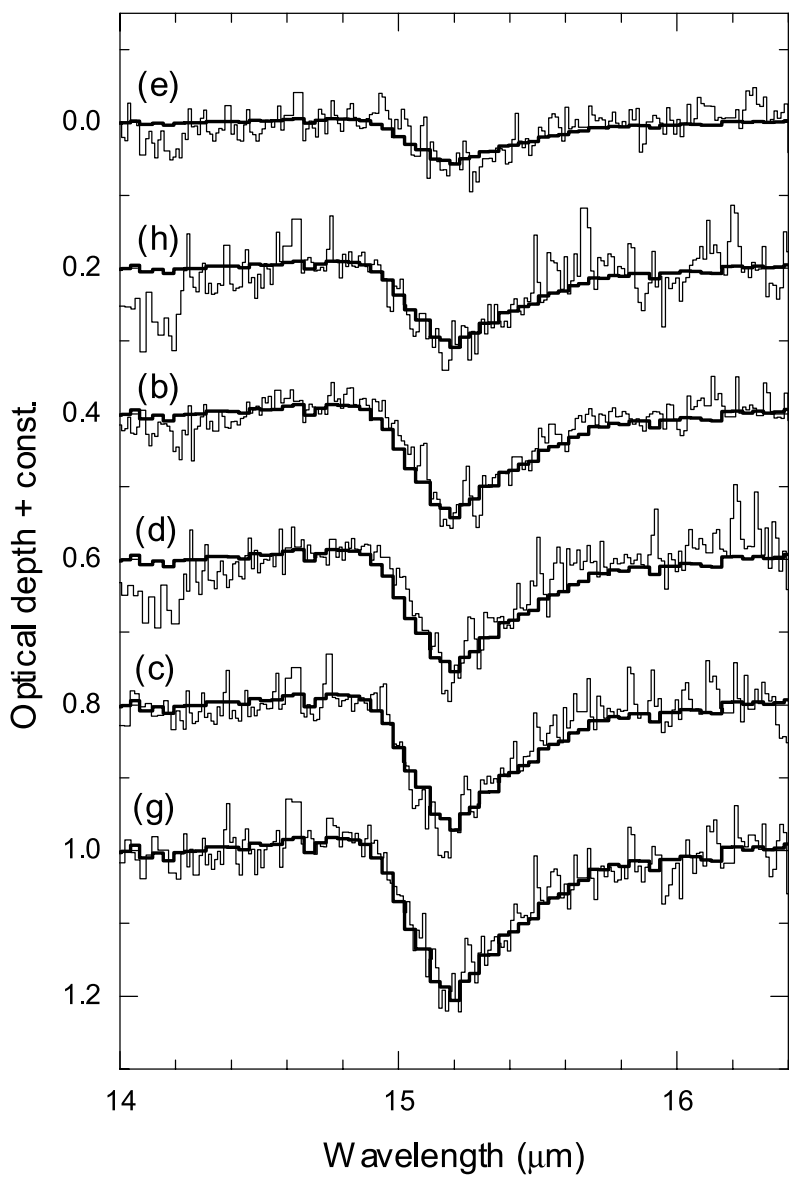

FIG. 2.-Optical depth spectra for six program stars with $\mathrm{CO}_{2}$ detections. The identification labels are the same as in Fig. 1 and Table 1. The plots are displaced vertically by an additive constant $(0.2 n ; n=0,1, \ldots, 5)$ for display and appear in order of increasing interstellar extinction from top to bottom. The thick line superposed on each plot is the E16 profile (Bergin et al. 2005) scaled to fit the spectrum. strength of the $\mathrm{CO}_{2}$-ice absorption in the optical depth spectra of these stars (Fig. 2) were evaluated with reference to the feature previously observed in the highly reddened Taurus field star Elias 16 (hereafter E16; Elias 1978; Bergin et al. 2005), which we adopt as a standard. The E16 profile is shown overlaid on the spectrum in Figure 2 for each line of sight in which we have a $\mathrm{CO}_{2}$ detection, smoothed to half its original resolution and scaled to match the depth of the feature in each case. Comparison indicates that the profile shape in each of these sources is identical to that in E16 to within the scatter inherent in the data. There is no clear evidence for structure that could be indicative of annealing. The E16 profile is well fitted by a two-component model based on laboratory spectra for polar $\left(\mathrm{H}_{2} \mathrm{O}: \mathrm{CO}_{2}\right)$ and apolar $\left(\mathrm{CO}: \mathrm{CO}_{2}\right)$ analogs at $10 \mathrm{~K}$, which contribute $85 \%$ and $15 \%$ of the total $\mathrm{CO}_{2}$ ice column density, respectively (see Bergin et al. 2005 for further details and discussion). We conclude that the profiles of the sources reported here are consistent with the same low-temperature combination to within the uncertainty. Although the data do not set very tight constraints on the detailed composition of the ice mixture, we can clearly exclude higher temperature $(T \approx 50-100 \mathrm{~K})$ ices such as those observed toward some YSOs (Gerakines et al. 1999; Watson et al. 2004).

We estimate the integrated optical depth $\left(\int \tau d \bar{\nu}\right)$ for each source with respect to its observed value in E16 $\left(5.0 \pm 0.1 \mathrm{~cm}^{-1}\right.$; Bergin et al. 2005) by evaluating the scaling constant that gives the best fit to the profile (Fig. 2). Results are listed in the right-hand column of Table 1, the error limits being determined by considering the highest and lowest values of the scaling constant deemed consistent with the data by visual inspection. The column density $N\left(\mathrm{CO}_{2}\right)$ is given by the ratio of the integrated optical depth to the band strength of the feature determined in the laboratory $\left(10^{-17} \mathrm{~cm}\right.$ per molecule; Gerakines et al. 1995). Results are listed in Table 2. Also included in Table 2 are data for major ice constituents $\left(\mathrm{H}_{2} \mathrm{O}\right.$ and $\mathrm{CO}$ as well as $\left.\mathrm{CO}_{2}\right)$ observed in these and other Taurus field stars, as available from the previous literature

TABLE 2

Column Densities of Ices toward Taurus Field Stars

\begin{tabular}{|c|c|c|c|c|c|c|c|}
\hline Source ID ${ }^{\mathrm{a}}$ & Association & Spectral Type ${ }^{\mathrm{b}}$ & $A_{V}$ & $N\left(\mathrm{H}_{2} \mathrm{O}\right)^{\mathrm{c}}$ & $N(\mathrm{CO})^{\mathrm{d}}$ & $N\left(\mathrm{CO}_{2}\right)$ & Reference \\
\hline 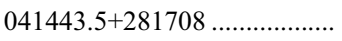 & & & $16.2 \pm 1.5$ & $\ldots$ & $\ldots$ & 2.7 & 1 \\
\hline $042324.6+250009$..................... & Elias 3 & K2 III & $10.0 \pm 0.5$ & 9.4 & 1.9 & 1.8 & 2 \\
\hline $042630.7+243637 \ldots \ldots \ldots \ldots \ldots \ldots$ & & $\ldots$ & $17.8 \pm 1.5$ & $16.4^{\mathrm{e}}$ & $7.4^{\mathrm{e}}$ & 3.0 & 1 \\
\hline $042930.2+265827 \ldots \ldots \ldots \ldots \ldots \ldots . .$. & & $\ldots$ & $11.4 \pm 1.5$ & $\ldots$ & $\ldots$ & 1.9 & 1 \\
\hline $043211.5+243338 \ldots \ldots \ldots \ldots \ldots \ldots$ & Elias 9 & M4 III & $4.9 \pm 0.5$ & 2.3 & $\ldots$ & $<0.5$ & 1 \\
\hline $043213.2+242910 \ldots \ldots \ldots \ldots \ldots \ldots . .$. & & $\ldots$ & $20.9 \pm 1.5$ & $22.5^{\mathrm{e}}$ & $7.5^{\mathrm{e}}$ & 3.6 & 1 \\
\hline 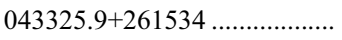 & Elias 13 & K2 III & $11.7 \pm 0.5$ & 10.8 & 1.3 & $1.5,2.1$ & 3,4 \\
\hline $043728.2+261024 \ldots \ldots \ldots \ldots \ldots \ldots$ & Tamura 2 & & $6.3 \pm 1.5$ & 4.0 & $\ldots$ & 1.0 & 1 \\
\hline $043926.9+255259 \ldots \ldots \ldots \ldots \ldots . .$. & Elias 15 & M2 III & $15.3 \pm 0.5$ & 15.0 & $4.1^{\mathrm{e}}$ & 2.5 & 1 \\
\hline 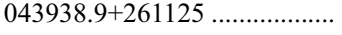 & Elias 16 & K3 III & $24.1 \pm 0.5$ & 25.7 & 6.5 & $5.0,5.8$ & 2,4 \\
\hline $044057.5+255413 \ldots \ldots \ldots \ldots \ldots \ldots$ & Tamura 8 & K5 III & $21.5 \pm 0.5$ & 24.7 & 6.0 & $\ldots$ & $\ldots$ \\
\hline $044124.7+255448 \ldots \ldots \ldots \ldots \ldots \ldots$ & HD 283809 & B3 V & $5.7 \pm 0.2$ & 2.0 & $\ldots$ & $<0.7$ & 1 \\
\hline $044401.7+252014$ & Tamura 17 & $\ldots$ & $4.0 \pm 1.5$ & 1.3 & $\ldots$ & $<0.1$ & 2 \\
\hline
\end{tabular}

Note.-All column densities are in units of $10^{17} \mathrm{~cm}^{-2}$.

${ }^{a}$ Format as in Table 1.

b Spectral type from Knez et al. (2005; Elias 16); Whittet et al. (1988; Tamura 8); SIMBAD database (HD 283809); and Elias (1978; all others).

${ }^{c}$ From published observations (Murakawa et al. 2000; Smith et al. 1993; Whittet et al. 1988) except where otherwise noted.

d From published observations (Chiar et al. 1995) except where otherwise noted. $N(\mathrm{CO})$ values were estimated by overlaying and scaling the observed E16 optical-depth profile in a manner consistent with that described in $\S 3$ for $\mathrm{CO}_{2}$; this resulted in minor differences for a few sources compared with values appearing in Chiar et al. (1995).

${ }^{\mathrm{e}}$ From unpublished spectra obtained in 2005 November with the Infrared Telescope Facility at Mauna Kea Observatory, Hawaii (J. E. Chiar et al. 2007, in preparation).

REFERENCES.- $N\left(\mathrm{CO}_{2}\right)$ : (1) this paper, Table 1; (2) Bergin et al. 2005; (3) Knez et al. 2005; (4) Nummelin et al. 2001. The Nummelin et al. results are from from ISO observations, and all other $\mathrm{CO}_{2}$ values are from Spitzer. 


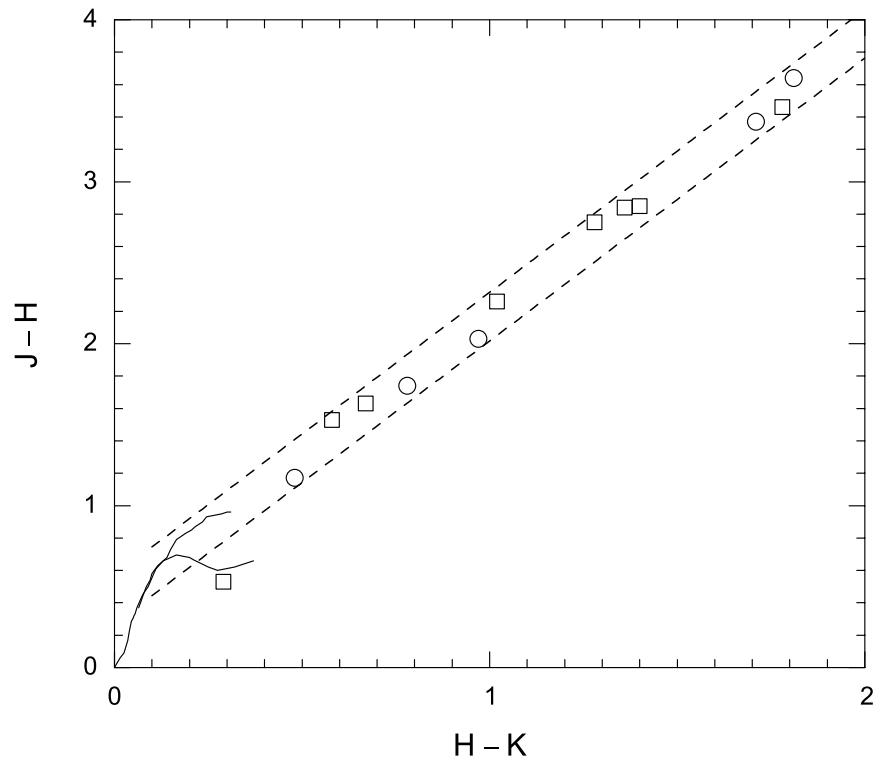

FIG. 3.-JHK color-color diagram for our program stars (squares) and the five additional stars listed in Table 2 (circles). Photometry is from 2MASS. Solid curves near the origin are intrinsic colors for normal unreddened stars of luminosity classes III and V (upper and lower branches). The dashed diagonal lines delimit the expected loci of reddened late-type giants for an extinction law $E_{J-H}=1.75 E_{H-K}$. The only object to plot outside this area of the diagram is HD $283809(J-H=0.53, H-K=0.29)$, an early-type star.

and new ground-based observations (see footnotes for references). The list includes all such lines of sight with $\mathrm{CO}_{2}$ detections or upper limits currently available. One object, Tamura 8, has no $\mathrm{CO}_{2}$ coverage at the present time, but is included to complete the set of solid CO detections in the cloud. For two stars with coverage of both the bending and stretching modes of $\mathrm{CO}_{2}$ (from Spitzer IRS and ISO SWS observations, respectively), differences between the resulting $N\left(\mathrm{CO}_{2}\right)$ values ( Table 2 ) are comparable with the uncertainties.

\section{EXTINCTION AND THE THRESHOLD EFFECT}

In this section we estimate interstellar reddening and extinction values for our program stars, and use the results to investigate the correlation of extinction with $N\left(\mathrm{CO}_{2}\right)$. Figure 3 shows the $J-H, H-K$ color-color diagram for the 13 stars in Table 2, using photometry from the Two Micron All Sky Survey (2MASS; Skrutskie et al. 2006). With the exception of HD 283809, which is an early-type star, all objects lie in a diagonal band consistent with normal intrinsic colors for late-type giants subject to a reddening displacement with mean slope

$$
E_{J-H} / E_{H-K}=1.75 \pm 0.05 \text {. }
$$

This reddening relation, obtained from a linear least-squares fit to the 12 points, is consistent with the average infrared extinction curve obtained by Indebetouw et al. (2005) for two regions of the Galactic plane (from which we estimate $E_{J-H} / E_{H-K}=1.73$ ).

For the early-type star HD 283809, we adopt the value of visual extinction $\left(A_{V}\right)$ calculated by Whittet et al. (2001), based on photometry in $B$ and $V$ as well as infrared passbands. The latetype stars in our sample lack $B V$ photometry, however, and $A_{V}$ must be estimated from infrared data alone. For stars with spectral type information available (Table 2), intrinsic colors were used to evaluate the color excesses $E_{J-H}$ and $E_{H-K}$ from the observed photometry (Bessell \& Brett 1988); for stars of unknown

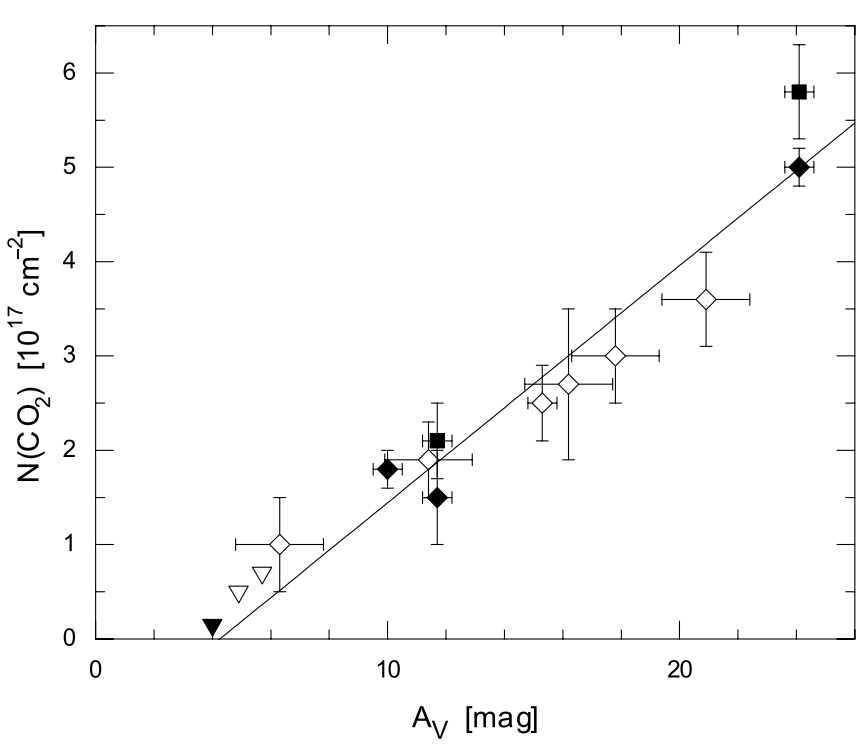

FIG. 4.- Plot of $\mathrm{CO}_{2}$ ice column density against visual extinction (Table 2). New results and values from previous literature are plotted as open and filled symbols, respectively. Inverted triangles denote upper limits. The filled squares are from $I S O$ observations of the $\mathrm{CO}_{2}$ stretching mode in two lines of sight, all other data are from Spitzer observations of the bending mode. The diagonal line is the weighted linear least-squares fit to all points excluding upper limits.

spectral type, these color excesses were estimated by considering the range of possible displacements parallel to the reddening vector onto intrinsic color lines in Figure 3. Visual extinction was then calculated from the relation

$$
A_{V}=5.2 E_{J-K}=5.2\left(E_{J-H}+E_{H-K}\right)
$$

where the factor $5.2( \pm 0.2)$ is the most probable value of the parameter $r$ (the ratio of total visual to selective infrared extinction) for the dense inner regions of the Taurus dark cloud (see Whittet et al. 2001; Whittet 2003). Results are listed in Table 2, errors reflecting uncertainty in the conversion factor (eq. [2]) and intrinsic colors (naturally most significant for those lacking spectral type information).

Figure 4 plots $\mathrm{CO}_{2}$ column density against extinction $\left(A_{V}\right)$ for stars in Table 2. The plot shows behavior analogous to that previously reported for $\mathrm{H}_{2} \mathrm{O}$ and $\mathrm{CO}$ ices (Whittet 2003; Whittet et al. 1988, 1989, 2001; Chiar et al. 1995), i.e., a rather high degree of positive correlation with nonzero intercept. The weighted linear least-squares fit (shown in Fig. 4) ignores limiting values; the fit yields a correlation coefficient of 0.97 and may be written in the form

$$
N\left(\mathrm{CO}_{2}\right)=q\left(A_{V}-A_{0}\right)
$$

where $q=(0.252 \pm 0.036) \times 10^{17} \mathrm{~cm}^{-2} \mathrm{mag}^{-1}$ is the slope and $A_{0}=4.3 \pm 1.0 \mathrm{mag}$ is the threshold extinction, i.e., the minimum visual extinction apparently required for detection of $\mathrm{CO}_{2}$ in the ices. Values of $A_{0}$ for other species in the same cloud are $3.2 \pm 0.1\left(\mathrm{H}_{2} \mathrm{O}\right)$ and $6.7 \pm 1.6(\mathrm{CO})$ [the corresponding values of $q$ are $1.30 \pm 0.04\left(\mathrm{H}_{2} \mathrm{O}\right)$ and $0.40 \pm 0.06(\mathrm{CO})$, in units of $\left.10^{17} \mathrm{~cm}^{-2} \mathrm{mag}^{-1}\right]$. The trend of increasing threshold extinction from $\mathrm{H}_{2} \mathrm{O}$ to $\mathrm{CO}_{2}$ to $\mathrm{CO}$ is consistent with the trend of increasing volatility, which would be expected if more volatile species require greater shielding from the external radiation field to remain 

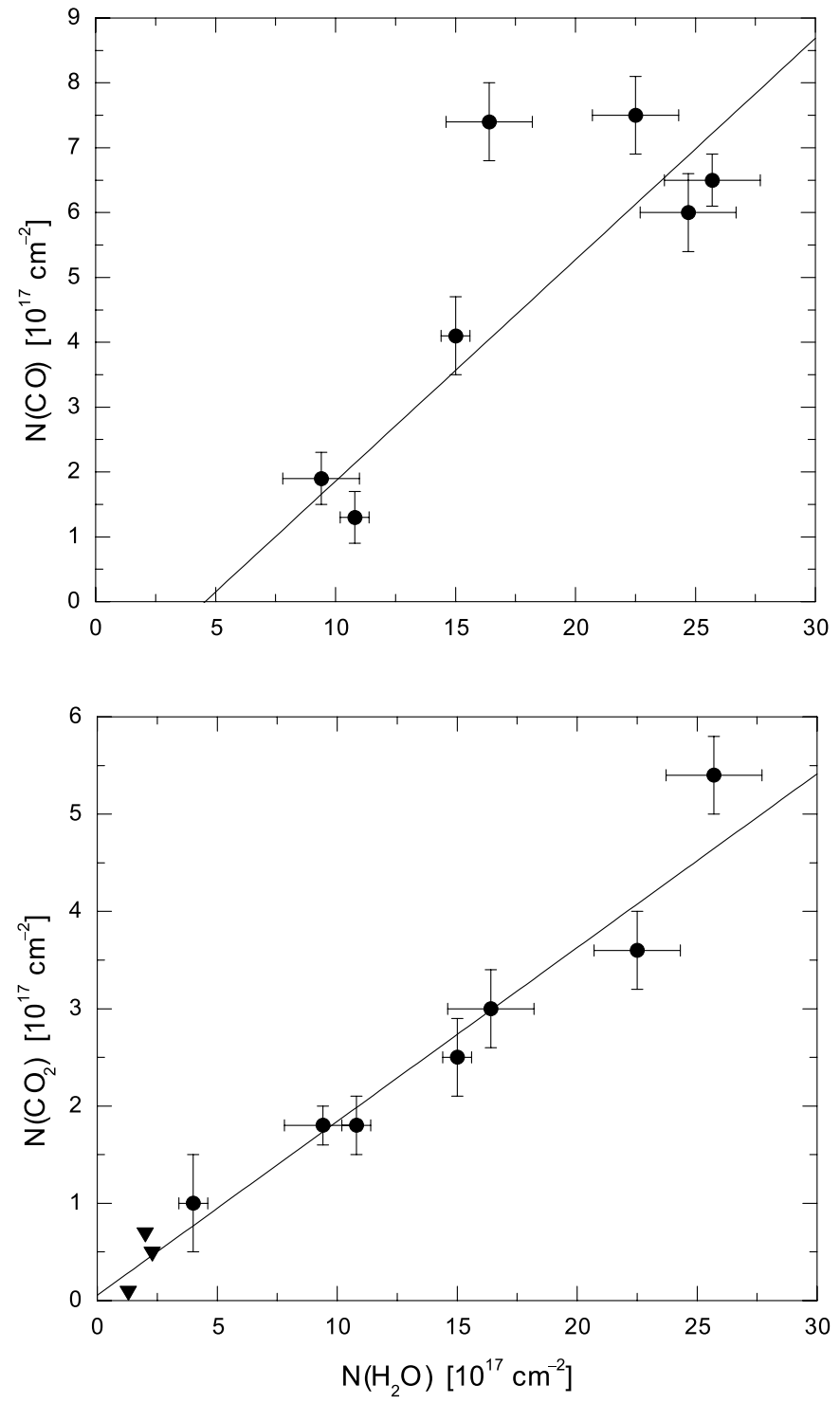

FIG. 5.- Plots of ice column density for $\mathrm{CO}_{2}$ (bottom panel) and $\mathrm{CO}$ (top panel ) against that for $\mathrm{H}_{2} \mathrm{O}$. ISO and Spitzer data for $\mathrm{CO}_{2}$ in the two relevant lines of sight ( Table 2) have been averaged. The weighted linear least-squares fit shown in each frame ignores limiting values (inverted triangles).

on the grains. The differences in $A_{0}$ are not large compared with the uncertainties, however.

\section{COLUMN DENSITY CORRELATIONS}

We next consider the intercorrelation of column densities in the ices. Figure 5 plots ${ }^{11} N\left(\mathrm{H}_{2} \mathrm{O}\right)$ versus $N\left(\mathrm{CO}_{2}\right)$ (bottom panel) and $N\left(\mathrm{CO}\right.$ ) (top panel) for all stars in Table $2 . \mathrm{As}_{2} \mathrm{O}$ is the dominant mantle constituent overall, $N\left(\mathrm{H}_{2} \mathrm{O}\right)$ may be regarded as a measure of the total column density of mantled grains. The $N\left(\mathrm{H}_{2} \mathrm{O}\right)$ versus $N\left(\mathrm{CO}_{2}\right)$ correlation is tight (correlation coefficient $\left.=0.99\right)$ and passes through the origin to within the uncertainty: the formal intercept on the $N\left(\mathrm{CO}_{2}\right)$ axis is $(0.06 \pm 0.55) \times 10^{17} \mathrm{~cm}^{-2}$. This result strongly supports the conclusion (Whittet et al. 1998; Bergin et al. 2005) that $\mathrm{H}_{2} \mathrm{O}$ and $\mathrm{CO}_{2}$ form in tandem, under essentially the same physical conditions, and coexist in the same ice compo-

\footnotetext{
${ }^{11}$ In this and subsequent figures, the $N\left(\mathrm{CO}_{2}\right)$ values obtained from observations of its bending and stretching modes (Spitzer and ISO, respectively; Table 2) have been averaged in the two relevant cases.
}

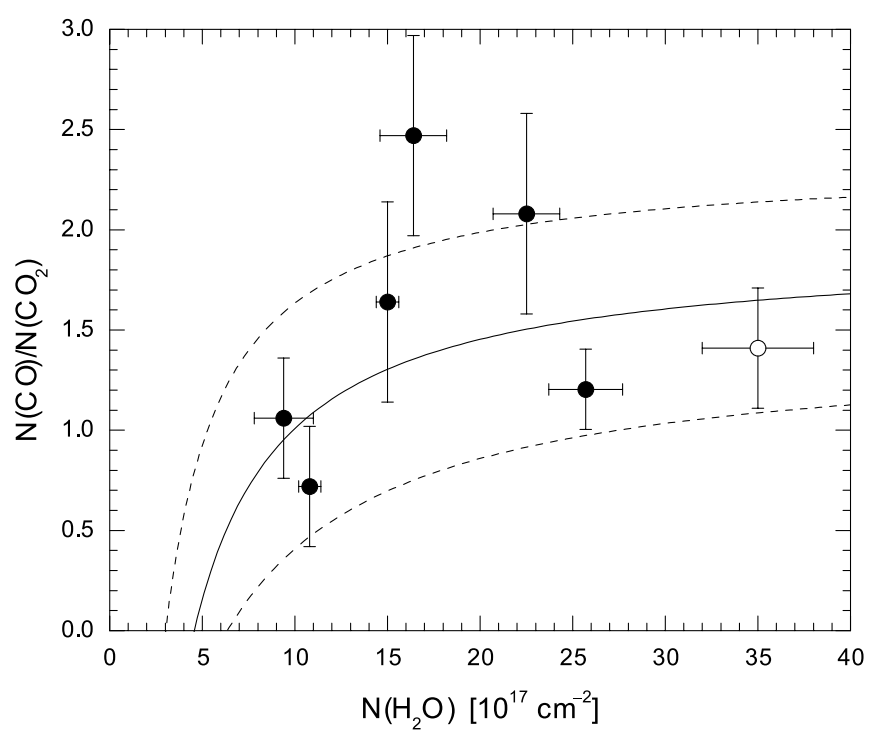

FIG. 6.-Plot of the column-density ratio $N(\mathrm{CO}) / N\left(\mathrm{CO}_{2}\right)$ vs. $N\left(\mathrm{H}_{2} \mathrm{O}\right)$. Data for Taurus field stars (Table 2) and the highly reddened field star CK2 in Serpens (Knez et al. 2005 and references therein; see $\S 6$ ) are plotted as filled and open circles, respectively. The solid curve is the trend predicted by ratioing the regression lines fitted to Taurus data in the two frames of Fig. 5; the dashed curves are approximate lower and upper bounds implied by the uncertainties in the fits.

nent. The correlation line, $N\left(\mathrm{CO}_{2}\right)=(0.18 \pm 0.04) N\left(\mathrm{H}_{2} \mathrm{O}\right)$, has a slope similar to values obtained previously for samples that include YSOs as well as field stars (Gerakines et al. 1999; Nummelin et al. 2001; Bergin et al. 2005), suggesting that the $\mathrm{CO}_{2}$ concentration is robust and not highly dependent on environment. If should be noted, however, that substantially higher $\mathrm{CO}_{2}$ concentrations, ranging up to approximately double that found here for the Taurus ICM, have been measured in the lines of sight to a few individual YSOs (see Nummelin et al. 2001; Boogert et al. 2004; Pontoppidan et al. 2005).

In contrast, the $N\left(\mathrm{H}_{2} \mathrm{O}\right)$ versus $N(\mathrm{CO})$ correlation (Fig. 5; top panel) has greater scatter (correlation coefficient $=0.88$ ) and a significant positive intercept $\left[(4.6 \pm 1.7) \times 10^{17} \mathrm{~cm}^{-2}\right]$ on the $N\left(\mathrm{H}_{2} \mathrm{O}\right)$ axis. This result implies that substantial accumulation of $\mathrm{CO}$ on the grains tends to occur only in situations where $\mathrm{H}_{2} \mathrm{O}$-rich mantles are already established. It is consistent with other evidence, based on the observed $\mathrm{CO}$ profile shape (Chiar et al. 1995), which indicates that, unlike $\mathrm{CO}_{2}$, the solid $\mathrm{CO}$ in Taurus resides primarily in an apolar component of the ices, segregated from the dominant $\mathrm{H}_{2} \mathrm{O}$-rich layer. The efficiency of $\mathrm{CO}$ depletion onto dust is a strong function of physical conditions (density and temperature), and the scatter in the $N\left(\mathrm{H}_{2} \mathrm{O}\right)$ versus $N(\mathrm{CO})$ plot presumably arises from the clumpy structure of the molecular gas (e.g., Langer et al. 1995).

To further illustrate the difference in behavior between $\mathrm{CO}$ and $\mathrm{CO}_{2}$ with respect to ice column, we plot in Figure 6 the ratio of $\mathrm{CO}$ to $\mathrm{CO}_{2}$ column density against $N\left(\mathrm{H}_{2} \mathrm{O}\right)$. The continuous curve is the empirical dependence resulting from the ratio of linear correlations for Taurus stars in the two frames of Fig. 5: i.e., for each $N\left(\mathrm{H}_{2} \mathrm{O}\right)$ value, the ratio is calculated from $N(\mathrm{CO})$ and $N\left(\mathrm{CO}_{2}\right)$ values predicted by the fits. The dashed curves in Figure 6 represent the approximate upper and lower bounds in this empirical dependence arising from the uncertainties in the fits. The plotted points include data for the object CK2 in Serpens (see $\S 6$ below) in addition to Taurus stars (Table 2). The trend suggested by the empirical curves is consistent with a scenario in which $\mathrm{CO}$ molecules attaching the grains at low ice columns $\left[N\left(\mathrm{H}_{2} \mathrm{O}\right)<\right.$ $4.6 \times 10^{17} \mathrm{~cm}^{-2}$ ] are rapidly oxidized to $\mathrm{CO}_{2}$ in tandem with 
$\mathrm{H}_{2} \mathrm{O}$ formation by hydrogenation of $\mathrm{O}$; at higher ice columns, $\mathrm{CO}$ becomes established in a separate mantle layer, reaching an overall concentration in the solid phase a factor of 1-2 times that of $\mathrm{CO}_{2}$.

\section{COMPARISON WITH THE SERPENS OBJECT CK2}

An important question to consider is whether the Taurus region studied here is representative of clouds with low- to intermediatemass star formation. Unfortunately, to date, no other comparable region has been observed in detail. A literature search identifies only one other confirmed field star, CK2 in the direction of the Serpens cloud (Churchwell \& Koornneef 1986), in which $\mathrm{H}_{2} \mathrm{O}$, $\mathrm{CO}$, and $\mathrm{CO}_{2}$ are all detected in solid form (Eiroa \& Hodapp 1989; Chiar et al. 1994; Knez et al. 2005). CK2 is classified K4 III (Knez et al. 2005), with visual extinction estimates ranging from $A_{V} \approx 34$ (Knez et al. 2005) to 46 or more (Chiar et al. 1994; Kaas et al. 2004). At 25-35 K, the mean ambient temperature of molecular gas in Serpens is higher than in typical dark clouds (White et al. 1995; McMullin et al. 2000), suggesting significant local heating of dust and gas by YSOs in the embedded cluster. Nevertheless, the abundance of solid $\mathrm{CO}$ toward to $\mathrm{CK} 2\left(\sim 40 \%\right.$ relative to $\mathrm{H}_{2} \mathrm{O}$, much of it residing in the more volatile apolar ice component; Chiar et al. 1994), implies that the line of sight must include dense regions where the grain temperature remains below $20 \mathrm{~K}$.

The three frames of Figure 7 compare CK2 (open circle) with Taurus data in column density intercorrelations for the available ices. Data for CK2 are taken from Knez et al. (2005) and references therein. In the case of $N\left(\mathrm{H}_{2} \mathrm{O}\right)$ the adopted value, which is based on ground-based observations of the $3.0 \mu \mathrm{m}$ stretching-mode absorption feature (Eiroa \& Hodapp 1989), is also consistent with Spitzer low-resolution IRS observations of the $6.0 \mu \mathrm{m}$ bending-mode feature (Knez et al. 2005; C. Knez 2006, personal communication). In general, CK2 does not follow the trends represented by extrapolation of the linear fits to Taurus data (Fig. 7, solid lines). Best agreement occurs in the case of $N\left(\mathrm{H}_{2} \mathrm{O}\right)$ versus $N(\mathrm{CO})$, for which a fit combining Taurus and Serpens data (dotted line) is also shown in Figure $7 b$. Departure from "Taurus-like" behavior is especially marked in the case of $N\left(\mathrm{H}_{2} \mathrm{O}\right)$ versus $N\left(\mathrm{CO}_{2}\right)$ : $\mathrm{CK} 2$ lies some $10 \sigma$ above the rather tight correlation line in Figure $7 a$. We conclude that ices toward CK2 must be overabundant in $\mathrm{CO}_{2}$ and/or underabundant in $\mathrm{H}_{2} \mathrm{O}$ compared with those in Taurus.

It is relevant to note that the $15 \mu \mathrm{m}$ solid $\mathrm{CO}_{2}$ profile in $\mathrm{CK} 2$ (Knez et al. 2005) is best fitted by a combination of ice mixtures qualitatively similar to those used to fit E16 (Bergin et al. 2005), i.e., with the contribution of $\mathrm{CO}_{2}$ in polar $\left(\mathrm{H}_{2} \mathrm{O}\right.$-rich $)$ ices dominating over that in apolar (CO-rich) ices. Based on this evidence, the expectation is that the $\mathrm{CO}_{2}$ column density should track that of $\mathrm{H}_{2} \mathrm{O}$ more closely than that of $\mathrm{CO}$, contrary to what is observed. However, the $15 \mu \mathrm{m}$ profile fit is not very sensitive to the $\mathrm{H}_{2} \mathrm{O}: \mathrm{CO}_{2}$ ratio of the polar component (for example, the 14:1 and 10:1 mixtures adopted by Bergin et al. and Knez et al., respectively, lead to similar fits). The ice composition toward CK2 might thus be explained by prior enhancement of the gas-phase $\mathrm{CO}$ abundance, leading, through surface chemistry $(\S 8)$, to a more $\mathrm{CO}_{2}$-rich polar phase, as well as to abundant CO in the apolar phase. The ambient UV radiation field from nearby embedded sources in Serpens might also come into play, driving efficient $\mathrm{CO} \rightarrow \mathrm{CO}_{2}$ conversion (e.g., Sandford \& Allamandola 1990).

\section{THE OXYGEN BUDGET IN THE INTRACLOUD MEDIUM}

Identification of the chemical forms assumed by elemental oxygen in interstellar clouds is important because the results constrain
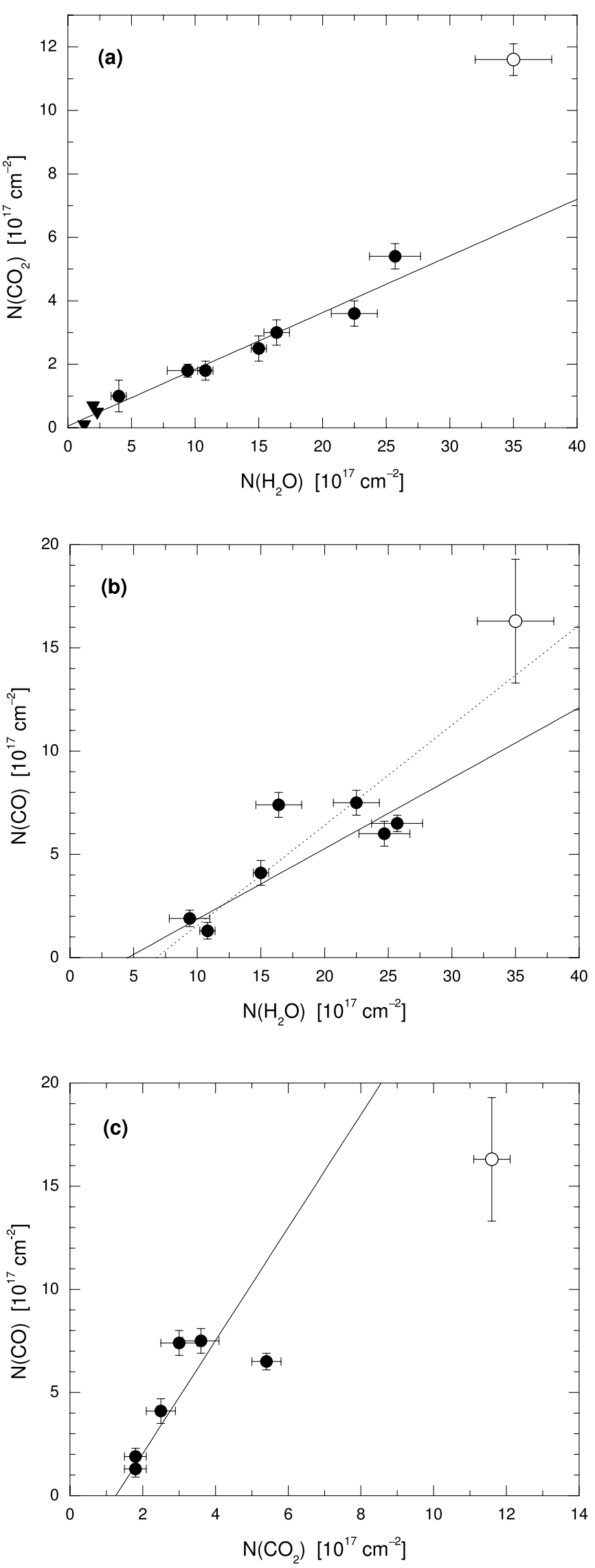

FIG. 7.-Comparison of ice column densities for Taurus ( filled circles; Table 2) and Serpens (open circles; $\S$ 6): (a) $\mathrm{H}_{2} \mathrm{O}$ vs. $\mathrm{CO}_{2} ;(b) \mathrm{H}_{2} \mathrm{O}$ vs. $\mathrm{CO}$; (c) $\mathrm{CO}_{2}$ vs. $\mathrm{CO}$. Solid lines are weighted linear least-squares fits to Taurus data only, ignoring upper limits (inverted triangles). In the case of $(b), \mathrm{H}_{2} \mathrm{O}$ vs. $\mathrm{CO}$, the data are consistent with a fit to all points (dotted line). 


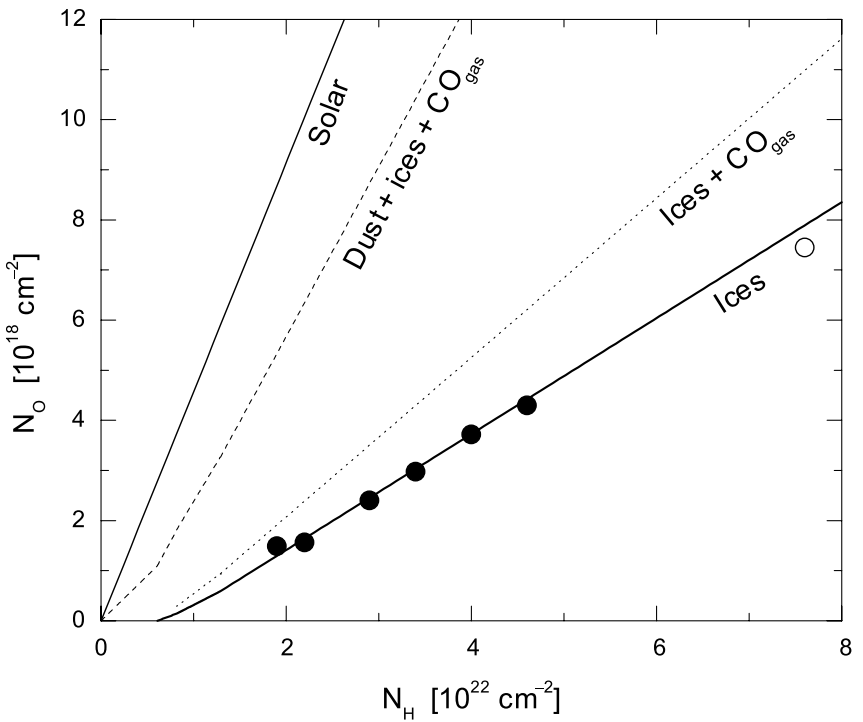

FIG. 8. - Plot of $N_{\mathrm{O}}$ vs. $N_{\mathrm{H}}$ for various potential oxygen reservoirs in the ISM. The thick solid line denotes the solar abundance, as reported by Asplund et al. (2004). The points are summed contributions to $\mathrm{O}$ in the ices observed toward Taurus and Serpens field stars ( filled and open circles). The solid and dotted curves are based on empirical correlations of ice $\left(\mathrm{H}_{2} \mathrm{O}, \mathrm{CO}\right.$, and $\left.\mathrm{CO}_{2}\right)$ and gas-phase $\mathrm{CO}$ column densities with extinction. The dashed curve includes the estimated contribution of refractory dust to $N_{\mathrm{O}}$. See the text $(\S 7)$ for detailed discussion.

models for the physics and chemistry of the interstellar medium (ISM; e.g., Bergin et al. 2000; Roberts \& Herbst 2002). This topic is currently controversial, as a consequence of tight observational limits set on the abundances of two potentially major oxygen reservoirs in molecular clouds, gas-phase $\mathrm{H}_{2} \mathrm{O}$ and $\mathrm{O}_{2}$ (e.g., Bergin \& Snell 2002; Goldsmith et al. 2000, 2002; Pagani et al. 2003). These observations naturally call into question the amount of oxygen that can be sequestered into dust grains and their icy mantles. A previous study (Vandenbussche et al. 1999) examined this question for a sample of YSOs observed by ISO. Our observations of major O-bearing ices toward background field stars with welldetermined extinction values in Taurus enable us to extend investigation of the oxygen budget to the intracloud medium. The results described below are summarized in Figure 8 and Table 3 .

We begin by assuming that the species included in our study $\left(\mathrm{H}_{2} \mathrm{O}, \mathrm{CO}\right.$, and $\left.\mathrm{CO}_{2}\right)$ provide a nearly-complete inventory of substantive oxygen reservoirs in the ices. Other possibilities include methanol $\left(\mathrm{CH}_{3} \mathrm{OH}\right)$, which is sometimes abundant in the envelopes of YSOs, but observations by Chiar et al. (1996) place an upper limit of $5 \%$ relative to $\mathrm{H}_{2} \mathrm{O}$ in ices toward Taurus field stars. $\mathrm{H}_{2} \mathrm{CO}, \mathrm{HCOOH}$, and OCS are also detected in YSOs but appear to be minor constituents of the ices at best, with abundances of no more than a few percent relative to $\mathrm{H}_{2} \mathrm{O}$ in any line of sight studied to date (e.g., Gibb et al. 2004). One potential exception is solid $\mathrm{O}_{2}$, but, unfortunately, its abundance cannot be readily quantified as it lacks sensitive infrared signatures: weak upper limits toward two YSOs (Vandenbussche et al. 1999) suggest that its contribution is no more than $\sim 75 \%$ of the $\mathrm{CO}_{2}$ contribution. With this caveat in mind, we estimate the column density of elemental $\mathrm{O}$ in ices toward our program stars from the equation

$$
N_{\mathrm{O}}(\text { ice })=N\left(\mathrm{H}_{2} \mathrm{O}\right)_{\text {ice }}+N(\mathrm{CO})_{\text {ice }}+2 N\left(\mathrm{CO}_{2}\right)_{\text {ice }} .
$$

Provided that gas and dust are well mixed in the ISM, the total hydrogen column density may be estimated from the extinction in the same lines of sight: the correlation

$$
N_{\mathrm{H}}=1.9 \times 10^{21} A_{V} \quad \mathrm{~cm}^{-2} \mathrm{mag}^{-1}
$$

is well established for lines of sight with relatively low $A_{V}$ that sample diffuse regions of the ISM (Bohlin et al. 1978; Whittet 1981). Extrapolation to denser, more opaque regions assumes that extinction increases in proportion to mass, notwithstanding any accompanying changes in the optical properties of the grains. Growth of the particles within dense clouds must affect their optical properties to some degree, but the two mechanisms for growth, coagulation, and mantle accretion, are predicted to have opposite effects: coagulation will tend to reduce the opacity per gram, whereas mantle growth will tend to increase it (Jura 1980; Kim \& Martin 1996; Whittet 2003). Given this ambiguity, we find no grounds for modifying the constant of proportionality in equation (5) at high $A_{V}$. In this respect, our analysis differs from that of Vandenbussche et al. (1999), who assumed a 70\% increase in $A_{V} / N_{\mathrm{H}}$.

We use equations (4) and (5) and the Taurus correlations for $N(\mathrm{X})_{\text {ice }}$ versus $A_{V}$ to generate the expected empirical relationship between $N_{\mathrm{O}}$ and $N_{\mathrm{H}}$, using data from this paper $(\S 4)$ for $\mathrm{X}=\mathrm{CO}_{2}$ and from the previous literature (Whittet et al. 1988, 1989, 2001; Chiar et al. 1995; Whittet 2003) for $\mathrm{X}=\mathrm{CO}$ and $\mathrm{H}_{2} \mathrm{O}$. Results are plotted in Figure 8 (line labeled "Ices"). Also

TABLE 3

\begin{tabular}{|c|c|c|c|c|c|}
\hline \multirow[b]{2}{*}{ SAMPLE } & \multirow[b]{2}{*}{ SPECIES } & \multicolumn{2}{|c|}{ AbUNDANCE $^{\mathrm{a}}$} & \multirow[b]{2}{*}{ REFERENCES $^{\mathrm{b}}$} & \multirow[b]{2}{*}{ Notes } \\
\hline & & $\mathrm{ppm}$ & $\%$ & & \\
\hline Solar abundance & & 457 & 100 & Asplund et al. (2004) & \\
\hline \multirow{2}{*}{ 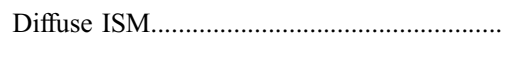 } & Atomic $\mathrm{O}$ & 319 & 70 & Meyer et al. (1998) & \\
\hline & Refractory dust & 138 & 30 & & 1 \\
\hline \multirow[t]{7}{*}{ Dense ICM } & Refractory dust & 138 & 30 & & 2 \\
\hline & Ices (total) & 116 & 26 & This work & \\
\hline & $\mathrm{H}_{2} \mathrm{O}$ ice & 68 & 15 & & \\
\hline & $\mathrm{CO}_{2}$ ice & 27 & 6 & & \\
\hline & $\mathrm{CO}$ ice & 21 & 5 & & \\
\hline & $\mathrm{CO}$ gas & 43 & 9 & Frerking et al. (1982) & 3 \\
\hline & Other (unkown) & 160 & 35 & & \\
\hline
\end{tabular}

Summary of OXYgEn Reservoirs IN THE ISM

${ }^{\text {a }}$ Abundances are expressed in parts per million (ppm) relative to $\mathrm{H}$ and percent relative to solar.

${ }^{\mathrm{b}}$ Notes: (1) It is assumed that all oxygen in the diffuse ISM not in atomic (OI) gas is tied up in refractory dust, consistent with the observed strength of interstellar silicate absorption (see text, $\S 7$ ). (2) The fraction of available $\mathrm{O}$ tied up in refractory dust in the dense intracloud medium is assumed to be the same as that in the diffuse ISM; i.e., no O-bearing refractory dust is created or destroyed in the transition between diffuse and dense environments. (3) Based on Fig. 9; see $\S 7$ for detailed discussion and caveats. 
plotted are points for individual stars that have data available for all three ice species. The good agreement between Serpens CK2 (open circle) and the Taurus relationship is probably fortuitous, given the uncertainty in $A_{V}$ and hence $N_{\mathrm{H}}$ for this source $(\S 6)$. The line intercepts the $N_{\mathrm{H}}$ axis at a value $\left(0.61 \times 10^{22} \mathrm{~cm}^{-2}\right)$ equivalent to the $\mathrm{H}_{2} \mathrm{O}$-ice threshold extinction $\left(A_{V}=3.2 \mathrm{mag}\right)$, the other species contributing to $N_{\mathrm{O}}$ (ice) as $N_{\mathrm{H}}$ increases beyond their respective thresholds. The total contribution of these ices to the oxygen budget within the cloud (ignoring the foreground component of $N_{\mathrm{H}}$ ) amounts to $\sim 116 \mathrm{ppm}$ relative to $\mathrm{H}$ (Table 3 ) or about a quarter of the solar abundance. ${ }^{12}$

We next consider the contribution of gaseous $\mathrm{CO}$ to the oxygen abundance within the Taurus cloud. Ideally, $N(\mathrm{CO})_{\text {gas }}$ should be evaluated from the strengths of interstellar absorption lines in the same continuum sources as the ices. Unfortunately, the only data available for relevant lines of sight are millimeterwave emission-line observations directed toward Taurus field stars from the Elias catalog (Frerking et al. 1982). As the cloud is $\sim 140 \mathrm{pc}$ from the Sun (Elias 1978) and $\sim 15^{\circ}$ below the Galactic equator, contamination of the beam by emission from material background to the cloud should be insignificant. Observations of rarer isotopic forms of $\mathrm{CO}$ were made to eliminate or minimize saturation. We have converted the resulting column densities to the common isotopic form using element isotope ratios for the local ISM from Wilson \& Rood (1994). ${ }^{13}$ Extinction values for the stars observed by Frerking et al. (1982) were recalculated using the method adopted in the current paper $(\S 4)^{14}$ and converted to $N_{\mathrm{H}}$ as above (eq. [5]). The results, plotted in Figure 9, suggest two distinct regimes of correlation, as first noted by Frerking et al., with a discontinuity at $N_{\mathrm{H}} \sim 0.9 \times 10^{22} \mathrm{~cm}^{-2}$ marking a sudden increase in $N(\mathrm{CO})_{\text {gas. }}$. It is interesting to note that this discontinuity corresponds to an extinction $\left(A_{V} \approx 4.7\right)$ close to the threshold value for $\mathrm{CO}_{2}$ ice detection $(\S 4)$, consistent with $\mathrm{CO}_{2}$ formation on grains driven by the availability of $\mathrm{CO}$ accreting from the gas. Some $30 \%-40 \%$ of the total CO appears to be depleted onto grains at high extinction (see also Chiar et al. 1995). We adopt the upper correlation line in Figure 9 as the most probable contribution of gas-phase $\mathrm{CO}$ to the oxygen budget in the Taurus cloud (that of the lower line is negligible). This is added to the ice component and plotted in Figure 8 (line labeled "Ices $+\mathrm{CO}_{\text {gas }}$ ").

Refractory solids contribute to the interstellar oxygen budget at all levels of density. The dominant O-rich form is silicate dust, identified spectroscopically by its infrared absorption features near 10 and $20 \mu \mathrm{m}$ : the strength of these features implies that essentially all of the available $\mathrm{Si}$ is bonded to $\mathrm{O}$ in this form (Mathis 1998). In diffuse regions devoid of ices or molecular gas, atomic $\mathrm{O}$ i gas accounts for the bulk of the available oxygen ( $319 \pm 14$ ppm; Meyer et al. 1998), and we assume that the remainder (138 ppm with respect to the solar value) is tied up in refractory dust. This assumption represents a good compromise between the minimum requirement of $\sim 100 \mathrm{ppm}$ in particles of generic composition $(\mathrm{Mg}, \mathrm{Fe}) \mathrm{SiO}_{3}$, needed to explain the strength of the silicate features, and the upper limit of $\sim 180 \mathrm{ppm}$ set by assuming that all metals are fully depleted into dust in their highest oxidation states (Meyer et al. 1998). Within quiescent

\footnotetext{
${ }^{12}$ We adopt the recent measurement of the solar oxygen abundance reported by Asplund et al. (2004) as the standard reference (Table 3). See also Asplund et al. (2005) for a recent review of the solar elemental composition.

${ }^{13}$ The adopted ratios were ${ }^{12} \mathrm{C} /{ }^{13} \mathrm{C}=77,{ }^{16} \mathrm{O} /{ }^{18} \mathrm{O}=560$, and ${ }^{16} \mathrm{O} /{ }^{17} \mathrm{O}=$ 1790.

${ }^{14}$ Our $A_{V}$ values are typically $\sim 13 \%$ higher than those quoted by Frerking et al. (1982), primarily owing to adoption of a different scaling factor in eq. (2).
}

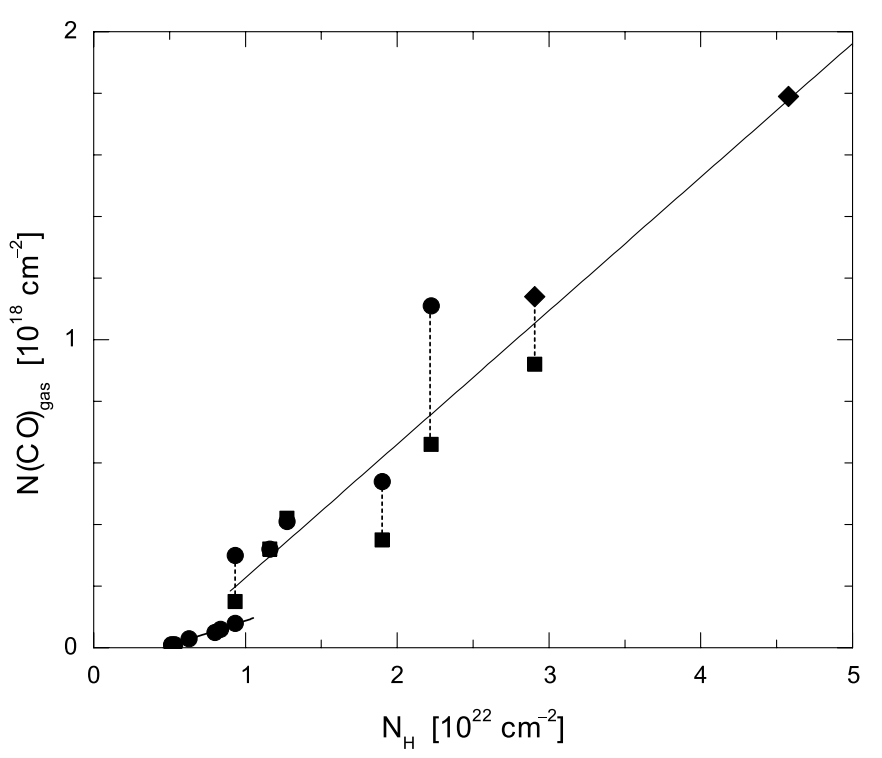

Fig. 9.-Plot of gas-phase $\mathrm{CO}$ column density toward Taurus field stars vs. total hydrogen column density. $\mathrm{CO}$ values are from observations (Frerking et al. 1982) of various isotopic forms: ${ }^{12} \mathrm{C}^{18} \mathrm{O}$ (circles), ${ }^{12} \mathrm{C}^{17} \mathrm{O}$ (squares), and ${ }^{13} \mathrm{C}^{18} \mathrm{O}$ (diamonds), converted to the common form using element isotope ratios for the local ISM from Wilson \& Rood (1994). Results from measurements of different isotopic forms in the same line of sight are joined by vertical dotted lines. The solid lines are linear least-squares fits to two distinct $N(\mathrm{H})$ ranges.

dense clouds, the refractory grains act as substrates for mantle growth but should otherwise survive without modification. We therefore assume the fraction of available $\mathrm{O}$ tied up in refractory dust in the dense ICM to be the same as in the diffuse ISM: the line labeled "Dust + ices $+\mathrm{CO}_{\text {gas }}$ " in Figure 8 includes this contribution. This summation accounts for $\sim 65 \%$ of the solar abundance within the cloud. See Table 3 for a summary of all results.

If the solar reference is a good standard for the ISM and our assumption of a constant $N_{\mathrm{H}} / A_{V}$ ratio is valid, then approximately $35 \%$ of the available oxygen abundance remains to be accounted for within the intracloud medium. In the diffuse ISM, observational constraints are, indeed, consistent with the full solar O-abundance split between gaseous atoms $(\sim 70 \%)$ and silicate dust $(\sim 30 \%)$, as noted above. In dense clouds, atomic $\mathrm{O}$ will naturally be converted to $\mathrm{H}_{2} \mathrm{O}, \mathrm{CO}, \mathrm{CO}_{2}, \mathrm{O}_{2}$ and other molecules by a variety of gasphase and grain-surface reactions (e.g., Roberts \& Herbst 2002), the products of which (with the partial exception of $\mathrm{CO}$ ) become depleted from the gas into icy mantles. Our observations set reasonably robust constraints on the ice component for the species considered, yet do not fully account for this conversion. Vandenbussche et al. (1999) reached a qualitatively similar conclusion for a sample of YSOs. Of their sources, R CrA IRS 2 appears to be the best proxy for the Taurus cloud, with a substantial apolar CO-ice component suggestive of minimal processing: we estimate from the quoted column densities that $\sim 52 \%$ of the oxygen is accounted for toward this source (ignoring upper limits, and after recalibration of the $N_{\mathrm{H}} / A_{V}$ ratio to eq. [5]), leaving a somewhat greater shortfall compared with our result.

Three possibilities are suggested for the identity of the "missing" oxygen:

1. Gas-phase CO.-The adopted abundance is quite uncertain as it could be affected both by sampling differences (comparing emission-line data with extinction estimates) and by saturation effects. $\mathrm{CO}$ abundances have been measured directly by absorptionline techniques toward some YSOs, yielding results that differ from each other by orders of magnitude (cf. Lacy et al. 1994; Shuping 
et al. 1999, 2001), presumably because of variable CO depletion/ sublimation driven by local conditions in the circumstellar envelopes. While the highest of these estimates might easily account for the missing oxygen, it seems unlikely to be a reliable guide to the situation within the quiescent intracloud medium.

2. Gas-phase atomic O.-Timescales for conversion of atomic $\mathrm{O}$ to molecular species in dark clouds are $\sim 10^{6} \mathrm{yr}$ according to the models of Roberts \& Herbst (2002). Dependent on the assumed age of the cloud, it may thus be reasonable to suppose that a significant fraction of the $\mathrm{O}$ remains in atomic form. Observations of cold cores along the line of sight to the Sgr B2 molecular cloud (Vastel et al. 2002) are consistent with a gas-phase $\mathrm{O} / \mathrm{CO}$ ratio of approximately 2.5 : if this result is applicable to the Taurus cloud it can account for most of the missing oxygen.

3. $\mathrm{O}_{2}$ ice.-This potentially important reservoir is not easily quantified, as previously discussed. The limit on the $\mathrm{O}_{2}$ abundance in solid form toward R CrA IRS 2 (Vandenbussche et al. 1999 ) is $<18 \mathrm{ppm}$, which suggests no more than a minor contribution to the missing oxygen. It would clearly be desirable to make a direct measurement of $\mathrm{O}_{2}$ in ices toward background field stars such as those studied in the present paper, but there is currently no observational facility capable of doing so.

\section{CONSTRAINTS ON CHEMICAL MODELS}

The presence of abundant $\mathrm{CO}_{2}$ in icy mantles within the quiescent intracloud regions of dark clouds issues a significant challenge to our understanding of interstellar chemistry (Tielens \& Whittet 1997; Whittet et al. 1998; Ruffle \& Herbst 2001; Roser et al. 2001; Stantcheva \& Herbst 2004). $\mathrm{CO}_{2}$ appears not to be produced in appreciable quantities by gas-phase chemical networks (van Dishoeck et al. 1996) unless shocks are present (Bergin et al. 1999), and is therefore assumed to form in situ on grain surfaces. The efficiency of production is critically dependent on the rates of the key surface reactions:

$$
\begin{gathered}
\mathrm{CO}+\mathrm{O} \rightarrow \mathrm{CO}_{2}, \\
\mathrm{CO}+\mathrm{OH} \rightarrow \mathrm{CO}_{2}+\mathrm{H}, \\
\mathrm{O}+\mathrm{HCO} \rightarrow \mathrm{CO}_{2}+\mathrm{H},
\end{gathered}
$$

of which at least the first two possess small activation barriers: see Ruffle \& Herbst (2001) for detailed discussion. These authors show that models with diffusive surface chemistry are capable of explaining the observed abundance of solid $\mathrm{CO}_{2}$ at reasonable temperatures and densities provided that diffusion of heavy species such as $\mathrm{O}$ is efficient.

A comparison between the observations discussed in this paper and representative photochemical models from Ruffle \& Herbst (2001) is shown in Figure 10, in which the solid-phase concentration of $\mathrm{CO}_{2}$ relative to $\mathrm{H}_{2} \mathrm{O}$ is plotted against that for CO. Observations for lines of sight in Taurus and Serpens are again plotted as filled and open circles, respectively. The vertical spread in the distribution for Taurus stars in Figure 10 is loosely correlated with extinction (highest toward the top). The models differ in that one assumes olivine grain cores at temperature $12 \mathrm{~K}$ (model B), the other amorphous carbon cores at $20 \mathrm{~K}$ (model D); both models assume a gas density $n_{\mathrm{H}}=2 \times 10^{4} \mathrm{~cm}^{-3}$ (see Ruffle $\&$ Herbst 2001 for further discussion and Fig. 6 of that paper for additional examples). Results are shown for various times in the evolution of the model cloud in each case. Figure 10 shows that an epoch exists for each model at which there is reasonable overlap with the observations: best agreement occurs for times $\sim 5 \times 10^{5} \mathrm{yr}$

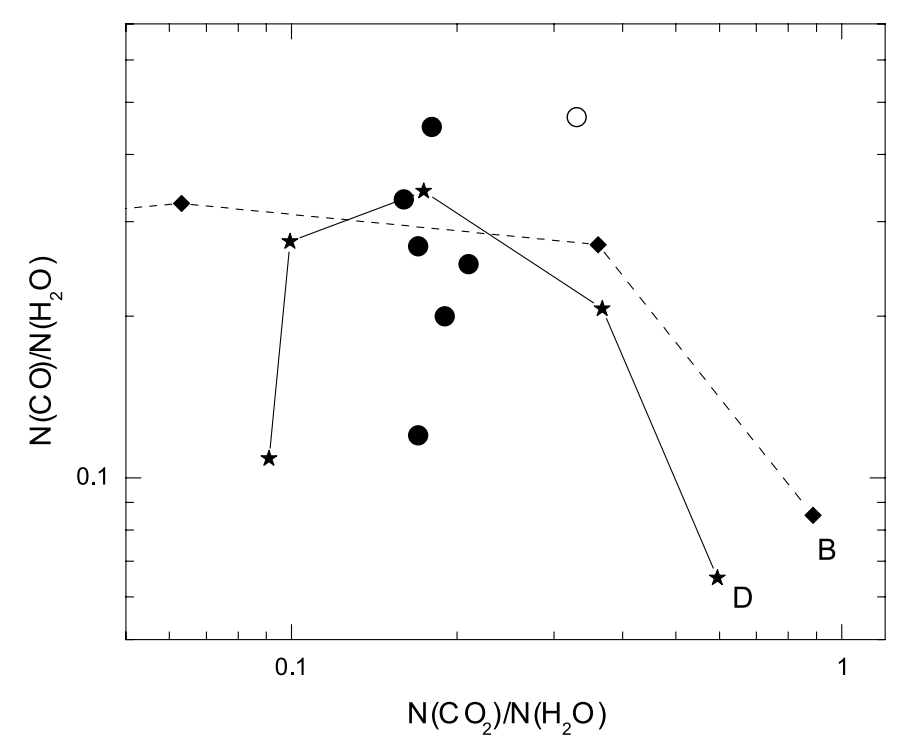

Fig. 10.-Plot of $\mathrm{CO}$ vs. $\mathrm{CO}_{2}$ concentrations relative to $\mathrm{H}_{2} \mathrm{O}$ in the ice mantles, comparing observational data (Taurus, filled circles; Serpens, open circle) with two representative photochemical models for gas-grain chemistry from Ruffle \& Herbst (2001). Model B (diamonds, dashed line) assumes olivine grain cores at temperature $12 \mathrm{~K}$, and model D (stars, solid line) assumes amorphous carbon cores at $20 \mathrm{~K}$; both models assume a gas density $n_{\mathrm{H}}=2 \times 10^{4} \mathrm{~cm}^{-3}$. Results are shown for various times (from cloud formation): the labeled point is for $t=10^{5} \mathrm{yr}$ for each model, and subsequent points from right to left are for $t=3 \times 10^{5}, 10^{6}, 3 \times 10^{6}$, and $10^{7} \mathrm{yr}$ (not all are shown for model B).

(model B) and $10^{6} \mathrm{yr}$ (model D) for Taurus (somewhat earlier for Serpens).

These timescales are sufficiently short to allow for an appreciable abundance of atomic $\mathrm{O}$ remaining in the gas (Roberts \& Herbst 2002), consistent with the proposal that this is an important reservoir of missing oxygen $(\S 7)$. Another question is then raised, however, as to how the $\mathrm{CO}$-rich, apolar component of the ices can form in a situation where atomic $\mathrm{O}$ remains abundant at all levels of cloud density: in such circumstances, it is expected that $\mathrm{CO}$ will be largely converted to $\mathrm{CO}_{2}$ (via reaction 6), whereas the observations (e.g., Fig. 6) indicate that $\mathrm{CO}$ is more abundant than $\mathrm{CO}_{2}$ in lines of sight with large ice columns. It may be the case that $\mathrm{O}_{2}$ becomes increasingly important as an oxygen reservoir (and $\mathrm{O}$ I correspondingly less important) as the density increases, a possibility that should be assessed in future theoretical work. Refinements of the chemical models are planned that will include updated energy parameters used in the reaction networks (E. Herbst 2006, personal communication). With these in place, it will be interesting to investigate, for example, whether the distribution in $N(\mathrm{CO}) / N\left(\mathrm{H}_{2} \mathrm{O}\right)$ for lines of sight in Taurus (Fig. 10) can be linked to physical conditions, and whether the offset between Taurus and Serpens implies a difference in evolutionary state.

\section{CONCLUSIONS}

The primary conclusions of this study may be summarized as follows:

1. Solid $\mathrm{CO}_{2}$ is routinely detected in the spectra of background stars that probe significant columns of interstellar dust within the Taurus dark cloud complex. The column density $N\left(\mathrm{CO}_{2}\right)$ correlates closely with extinction and displays a threshold effect similar to that seen previously for other species in the ices.

2. $N\left(\mathrm{CO}_{2}\right)$ displays a tight correlation with $N\left(\mathrm{H}_{2} \mathrm{O}\right)$ that passes through the origin to within observational error. This supports 
an earlier conclusion that these species form in tandem under the same physical conditions and coexist in the same (polar) ice layer on the grains.

3. The behavior of $N(\mathrm{CO})$ relative to $N\left(\mathrm{CO}_{2}\right)$ is consistent with a scenario in which $\mathrm{CO}$ molecules attaching the grains at low ice columns $\left[N\left(\mathrm{H}_{2} \mathrm{O}\right)<4.6 \times 10^{17} \mathrm{~cm}^{-2}\right]$ are rapidly oxidized to $\mathrm{CO}_{2}$ in tandem with $\mathrm{H}_{2} \mathrm{O}$ formation by hydrogenation of $\mathrm{O}$; at higher ice columns, $\mathrm{CO}$ becomes established in a separate (apolar) layer.

4. Differences in ice composition between lines of sight toward the Taurus and Serpens clouds are consistent with enhanced production of $\mathrm{CO}_{2}$ in the latter. This might result from differences in physical conditions and/or evolutionary state between the two clouds.

5. Our results are used to investigate the distribution of elemental oxygen between potential reservoirs in the intracloud medium. We show that $\sim 65 \%$ of the solar $\mathrm{O}$ abundance can be accounted for by summing the contributions of ice mantles, refractory dust, and gas-phase CO. Candidates for the remainder include atomic $\mathrm{O}_{\mathrm{I}}$ gas and solid $\mathrm{O}_{2}$. It is also possible that the contribution of gaseous $\mathrm{CO}$ has been significantly underestimated.

6. The observed composition of the mantles is consistent with the predictions of photochemical models with diffusive surface chemistry, based on the assumption that heavy species such as $\mathrm{O}$ diffuse efficiently.
It will be important, in the future, to obtain observations that allow a more detailed comparison between Taurus and other clouds than is possible here using the one available line of sight in Serpens. Such comparisons are especially important as the means of establishing the range in ice composition that can exist within clouds, thus placing additional constraints on chemical models. These studies are a prerequisite for reliable interpretation of ice features in YSO spectra, where ices originating in the intracloud medium may be subject to local modification through exposure to radiation fields, winds and shocks driven by the embedded sources.

This work is based on observations made with the Spitzer Space Telescope, which is operated by the Jet Propulsion Laboratory, California Institute of Technology, under a contract with NASA. Financial support for this work was provided by NASA through an award issued by JPL/Caltech (Support Agreement no. 1264149). We are grateful to Dan Watson for advice on IRS data reductions, and to Eric Herbst and an anonymous referee for constructive comments on the manuscript. D. C. B. W. also wishes to thank Stefi Baum and Joel Kastner for hospitality and support during his sabbatical leave at Rochester Institute of Technology.

\section{REFERENCES}

Asplund, M., Grevesse, N., \& Sauval, A. J. 2005, in ASP Conf. Ser. 336, Cosmic Abundances as Records of Stellar Evolution and Nucleosynthesis, ed. F. N. Bash \& T. G. Barnes (San Francisco: ASP), 25

Asplund, M., Grevesse, N., Sauval, A. J., Allende Prieto, C., \& Kiselman, D. 2004, A\&A, 417, 751

Bergin, E. A., Melnick, G. J., Gerakines, P. A., Neufeld, D. A., \& Whittet, D. C. B. 2005, ApJ, 627, L33

Bergin, E. A., Neufeld, D. A., \& Melnick, G. J. 1999, ApJ, 510, L145

Bergin, E. A., \& Snell, R. L. 2002, ApJ, 581, L105

Bergin, E. A., et al. 2000, ApJ, 539, L129

Bessell, M. S., \& Brett, J. M. 1988, PASP, 100, 1134

Bohlin, R. C., Savage, B. D., \& Drake, J. F. 1978, ApJ, 224, 132

Boogert, A. C. A., et al. 2004, ApJS, 154, 359

Chiar, J. E., Adamson, A. J., Kerr, T. H., \& Whittet, D. C. B. 1994, ApJ, 426, 240

. 1995, ApJ, 455, 234

Chiar, J. E., Adamson, A. J., \& Whittet, D. C. B. 1996, ApJ, 472, 665

Chiar, J. E., et al. 2000, ApJ, 537, 749

Churchwell, E., \& Koornneef, J. 1986, ApJ, 300, 729

Dartois, E., Demyk, K., d’Hendecourt, L., \& Ehrenfreund, P. 1999, A\&A, 351, 1066

Dartois, E., Pontoppidan, K., Thi, W.-F., \& Muñoz Caro, G. M. 2005, A\&A, 444, L57

Decin, L., Morris, P. W., Appleton, P. N., Charmandaris, V., Armus, L., \& Houck, J. R. 2004, ApJS, 154, 408

de Graauw, Th., et al. 1996, A\&A, 315, L345

d'Hendecourt, L., et al. 1996, A\&A, 315, L365

Ehrenfreund, P., et al. 1999, A\&A, 350, 240

Eiroa, C., \& Hodapp, K. W. 1989, A\&A, 210, 345

Elias, J. H. 1978, ApJ, 224, 857

Frerking, M. A., Langer, W. D., \& Wilson, R. W. 1982, ApJ, 262, 590

Gerakines, P. A., Schutte, W. A., Greenberg, J. M., \& van Dishoeck, E. F. 1995 , A\&A, 296, 810

Gerakines, P. A., et al. 1999, ApJ, 522, 357

Gibb, E. L., Whittet, D. C. B., Boogert, A. C. A., \& Tielens, A. G. G. M. 2004, ApJS, 151, 35

Goldsmith, P. F., et al. 2000, ApJ, 539, L123 2002, ApJ, 576, 814

Higdon, S. J. U., et al. 2004, PASP, 116, 975

Houck, J. R., et al. 2004, ApJS, 154, 18

Indebetouw, R., et al. 2005, ApJ, 619, 931

Jura, M. 1980, ApJ, 235, 63

Kaas, A. A., et al. 2004, A\&A, 421, 623

Kim, S.-H., \& Martin, P. G. 1996, ApJ, 462, 296
Knez, C., et al. 2005, ApJ, 635, L145

Lacy, J. H., Knacke, R., Geballe, T. R., \& Tokunaga, A. T. 1994, ApJ, 428, L69 Langer, W. D., Velusamy, T., Kuiper, T. B. H., Levin, S., Olsen, E., \& Migenes, V. 1995, ApJ, 453, 293

Mathis, J. S. 1998, ApJ, 497, 824

McMullin, J. P., Mundy, L. G., Blake, G. A., Wilking, B. A., Mangum, J. G., \& Latter, W. B. 2000, ApJ, 536, 845

Meyer, D. M., Jura, M., \& Cardelli, J. A. 1998, ApJ, 493, 222

Murakawa, K., Tamura, M., \& Nagata, T. 2000, ApJS, 128, 603

Nummelin, A., Whittet, D. C. B., Gibb, E. L., Gerakines, P. A., \& Chiar, J. E. 2001, ApJ, 558, 185

Pagani, L., et al. 2003, A\&A, 402, L77

Pontoppidan, K. M., et al. 2005, ApJ, 622, 463

Roberts, H., \& Herbst, E. 2002, A\&A, 395, 233

Roser, J., Vidali, G., Giulio, M., \& Pirronello, V. 2001, ApJ, 555, L61

Ruffle, D. P., \& Herbst, E. 2001, MNRAS, 324, 1054

Sandford, S. A., \& Allamandola, L. J. 1990, ApJ, 355, 357

Shuping, R. Y., Chiar, J. E., Snow, T. P., \& Kerr, T. 2001, ApJ, 547, L161

Shuping, R. Y., Snow, T. P., Crutcher, R., \& Lutz, B. L. 1999, ApJ, 520, 149

Skrutskie, M. F., et al. 2006, AJ, 131, 1163

Smith, R. G., Sellgren, K., \& Brooke, T. Y. 1993, MNRAS, 263, 749

Stantcheva, T., \& Herbst, E. 2004, A\&A, 423, 241

Tielens, A. G. G. M., \& Whittet, D. C. B. 1997, in IAU Symp. 178, Molecular Astrophysics: Probes and Processes, ed. E. F. van Dishoeck (Dordrecht: Kluwer), 45

van Broekhuizen, F. A., Groot, I. M. N., Fraser, S. A., van Dishoeck, E. F., \& Schlemmer, S. 2006, A\&A, 451, 723

Vandenbussche, B., et al. 1999, A\&A, 346, L57

van Dishoeck, E. F., et al. 1996, A\&A, 315, L349

Vastel, C., Polehampton, E. T., Baluteau, J.-P., Swinyard, B. M., Caux, E., \& Cox, P. 2002, ApJ, 581, 315

Watson, D. M., et al. 2004, ApJS, 154, 391

White, G. J., Casali, M. M., \& Eiroa, C. 1995, A\&A, 298, 594

Whittet, D. C. B. 1981, MNRAS, 196, 469

2003, Dust in the Galactic Environment (2nd ed; Bristol: IOP)

Whittet, D. C. B., Adamson, A. J., Duley, W. W., Geballe, T. R., \& McFadzean, A. D. 1989 , MNRAS, 241, 707

Whittet, D. C. B., Bode, M. F., Longmore, A. J., Adamson, A. J., McFadzean, A. D., Aitken, D. K., \& Roche, P. F. 1988, MNRAS, 233, 321

Whittet, D. C. B., Gerakines, P. A., Hough, J. H., \& Shenoy, S. S. 2001, ApJ, 547,872

Whittet, D. C. B., et al. 1998, ApJ, 498, L159

Wilson, T. L., \& Rood, R. T. 1994, ARA\&A, 32, 191 